\title{
Military and VA telemedicine systems for patients with traumatic brain injury
}

\author{
Philip Girard, MS \\ Defense and Veterans Brain Injury Center, Walter Reed Army Medical Center, Washington, DC; Manchester Depart- \\ ment of Veterans Affairs Medical Center, Manchester, NH
}

\begin{abstract}
Telemedicine plays a critical role within the Department of Veterans Affairs (VA) Veterans Health Administration by allowing the surveillance and care of patients who are isolated by geography, poverty, and disability. In military settings, telemedicine is being widely used to identify injury and illness and aid in the treatment, rehabilitation, and recovery of combat-wounded soldiers in theater. Rapid advances in both domains are transforming the way clinicians provide care, education, and support to patients with traumatic brain injury (TBI) and their families. This article discusses the military and VA telemedicine capabilities that are supporting the care of service members and veterans with TBI. These capabilities include new technologies that enhance the identification of TBI, management of symptoms in theater, and application of proven technologies (interactive video, Internet, and World Wide Web) to improve overall care coordination throughout military and VA systems. The impact of distance learning, teleconsultation, telerehabilitation, and home telehealth programs is also described within this context.
\end{abstract}

Key words: brain injury, care coordination, concussion assessment, distance learning, home healthcare, rehabilitation, teleconsultation, telehealth, telemedicine, telerehabilitation.

\section{INTRODUCTION}

Rapid advances in medical technology are transforming the way clinicians provide care, education, and support to patients and families in remote locations. Using technology to provide medical consultation and treatment over distance is known as telemedicine. Telemedicine plays a critical role within the Department of Veterans Affairs (VA) Veterans Health Administration (VHA) by allowing the surveillance and care of patients who are isolated by geography, poverty, and disability. Veterans once at risk of being left untreated can now be monitored and cared for in their homes and communities. VHA home telehealth programs are reducing hospitalizations, emergency room visits, and length of hospital stays while improving the quality of life for veterans [1]. In military settings, telemedicine is being widely used to identify

\footnotetext{
Abbreviations: ANAM = Automated Neuropsychological Assessment Metrics, DOD = Department of Defense, DVBIC = Defense and Veterans Brain Injury Center, LOC $=$ loss of conciousness, $\mathrm{MS}=$ multiple sclerosis, $\mathrm{OCC}=$ Office of Care Coordination, $\mathrm{PA}=$ physician's assistant, $\mathrm{PCS}=$ postconcussion syndrome, $\mathrm{PDA}=$ personal data assistant, $\mathrm{PTA}=$ posttraumatic amnesia, PTSD = posttraumatic stress disorder, SCI = spinal cord injury, TBI = traumatic brain injury, VA = Department of Veterans Affairs, VAMC = VA medical center, VHA = Veterans Health Administration, VISN = Veterans Integrated Service Network, VTC = video-teleconferencing, WRAMC = Walter Reed Army Medical Center.

Address all correspondence to Philip Girard, MS; Defense and Veterans Brain Injury Center, Office of Telemedicine, Manchester VAMC, 718 Smyth Rd, Manchester, NH 03103; 603-617-0293; fax: 603-626-6502.

Email: philip.girard@va.gov

DOI: 10.1682/JRRD.2006.12.0174
} 
injury and illness and aid in the treatment, rehabilitation, and recovery of combat-wounded soldiers in theater. Interactive video-teleconferencing (VTC) and Web-based "store-and-forward" technology are bridging the gap between doctors and patients separated by hostile combat environments. The military's use of telemedicine provides patients greater access to specialized medical services and thereby improves the continuity of care for critically wounded patients while preventing unnecessary medical evacuations that reduce unit readiness [2].

Model programs in both VA and military settings [1-2] have demonstrated how technology can enhance traumatic brain injury (TBI) identification, recovery, and support. Like many telemedicine services, these capabilities were originally developed around a particular clinical discipline and then later adopted for use in other areas. The use of telemedicine to deliver psychosocial and community-based nursing services, for example, began as a way to help the elderly live more independently at home. Over time, the VHA adapted specific aspects of "telehealth" programs to improve the care of aging veterans with disabilities, including those with TBI.

The increase in combat casualties with TBI from current conflicts has spurred demand for telemedicine solutions that can extend such clinical activities as neurological assessment, acute medical and neurosurgical treatment, psychiatric intervention, behavioral therapies, occupational and physical rehabilitation, and overall service coordination between military, VA, and community programs. The heterogeneous nature of TBI requires the cooperation of many different disciplines and services and, thus, patients who sustain brain injuries may benefit from multiple forms of telemedicine over the course of their care.

As medical technology enters an age of surgical robotics [3] and thought-controlled prosthetics [4], even more capabilities will become available to soldiers and veterans with TBI and their families. Bold, yet often simple, ideas can hold great promise for those injured in combat and in civilian life. Transferring these telemedicine capabilities to other sectors of healthcare will improve the entire continuum of TBI care and recovery.

\section{TRAUMATIC BRAIN INJURY}

A basic knowledge of brain injury severity is needed to understand the interplay between clinical services and telemedicine. Briefly, severity of TBI is highly variable and can be described in many ways but is typically categorized as mild, moderate, or severe. In mild TBI, a brief change in mental status or consciousness occurs that may be followed by temporary symptoms associated with concussion, such as headache, blurred vision, and confusion [5]. The symptoms of mild TBI generally resolve quickly, but optimal recovery may require patients to temporarily limit their activity to prevent further injury or harm to others.

In moderate TBI, longer periods of loss of consciousness (LOC) and posttraumatic amnesia (PTA) follow the traumatic event. Patients with moderate TBI may experience a variety of symptoms, including mood and memory disturbances and physical and emotional problems that may persist for months. Moderate TBI requires increased clinical intervention but generally responds to medication, psychotherapy, and compensatory strategies [6].

Severe TBI can be characterized by a coma that lasts longer than 24 hours or PTA for longer than 7 days. The Glasgow Coma Score can also be used to describe the severity of TBI, with severe patients falling between 3 and 8 on the scale. Patients with severe TBI may remain in a vegetative state for an extended time period. Severe TBI most often results in long-term problems with independent functioning and can result in moderate to severe disability in some patients. Most patients with severe TBI require comprehensive nursing care and ongoing rehabilitation.

The challenges involved in managing TBI exist on many levels. First, the unique occurrence of physical, cognitive, and emotional symptoms associated with TBI requires the cooperation of many disciplines. Second, severe TBI frequently occurs with other traumatic injuries that can complicate emergency treatment, recovery, and rehabilitation. Third, mild and moderate TBI, particularly for those in which the head is not penetrated, may not be immediately obvious. Symptoms can be subtle. Injuries can occur in isolation and are sometimes repeated, especially in combat situations. Together, these factors add to the difficulty of early identification of mild to moderate TBI.

\section{TRAUMATIC BRAIN INJURY IDENTIFICATION AND ASSESSMENT}

With blasts contributing to high numbers of head injuries in theater (Operation Enduring Freedom/Operation 
Iraqi Freedom) [7], an increased pressure is on medics to quickly assess and treat TBI. However, the limited time and resources available at forward medical commands prohibit thorough TBI evaluation, which often results in service members returning from duty with symptoms of undiagnosed TBI. These symptoms can include fatigue, irritability, depression, concentration problems, and sensitivity to noise and light. Such problems can affect reaction time and decision making, which are critical in maintaining the performance and safety of soldiers on duty. Further, undiagnosed TBI increases the risk of secondary injury and may pose a risk of harm to others, especially in military populations.

Recognizing the need for improved TBI identification, researchers at the Defense and Veterans Brain Injury Center (DVBIC) have tested a remote cognitive assessment system that may allow clinicians in the field to more rapidly gather information on reaction time, memory, and mood. When combined with other medical information, these data present a more complete picture of a patient's cognitive and emotional status. Using secure Web-based systems, TBI specialists at distant sites can review this information and, working with forward medical staff, develop treatment strategies and return-to-duty recommendations.

The goal of the DVBIC research is to test the technical feasibility of remote cognitive assessment systems that could (1) allow medics to identify TBI and postconcussion syndrome (PCS) in the field and (2) give VA and community healthcare providers the ability to identify TBI remotely. The TBI assessment system is a Webbased program that contains evaluation questionnaires and brief cognitive screening tests, including-

1. The PCS Checklist and Scale, which assess the most common symptoms experienced after TBI.

2. The State-Trait Anxiety Inventory and the Automated Neuropsychological Assessment Metrics (ANAM) Mood and Sleep Scales, which provide focused assessment of mood and anxiety disturbance.

3. The DVBIC clinical tracking form, which documents injury characteristics such as severity and cause.

4. The ANAM Simple Reaction Time and Continuous Performance subtests, which objectively measure cognitive functioning.

Together these tests help clinicians identify the areas of the brain that may have been affected by the injury. Step-by-step instructions are provided as the patient works through the test. Areas of concern can be flagged for easy reference by physician's assistants (PAs) onsite, and full reports can be gathered by a neuropsychologist at different intervals or reviewed by specialists and returned with recommendations. Various technical, security, and logistical issues are involved in the delivery of such testing; however, limited deployment with a laptop computer with high-speed Internet access has been successful.

Computerized cognitive assessment becomes even more useful when baseline test data (i.e., before the head trauma) are available. Specifically, the effects of concussion (mild TBI) can be better determined by comparing pre- and postinjury performance. This has been documented in sports concussions, where high school and college football players were evaluated preseason and then again after sustaining physical impacts on the playing field [8]. Testing with a computerized battery known as ImPACT (http://www.impacttest.com/) revealed cognitive deficits in asymptomatic athletes within 4 days postconcussion. Although physical effects (dizziness, headache, nausea, etc.) were not reported, cognitive domains (reaction time, processing speed, and memory) were significantly diminished and only gradually returned to normal over several days and weeks [8]. Such testing also supports the conclusion that prior concussion increases the risk of subsequent head injury and that repeat concussions result in more significant cognitive deficits [9]. Developing reliable tests that are easy to administer has become increasingly important for all these reasons.

Computerized tests offer several advantages over paper-and-pencil methods, including the ability to (1) reliably measure reaction time (to $1 / 100$ th of a second), (2) reduce practice effects by presenting alternate forms and questions, and (3) immediately score and communicate results [8]. Additionally, handheld data recording devices now allow sports medicine professionals to administer cognitive tests from the sidelines immediately after the athlete sustains a concussion. This added portability further increases the appeal of computerized systems that can now be administered in other environments.

Unfortunately, in combat environments, tactical situations usually prohibit cognitive testing early on, so more routine assessment measures have also been developed. For example, sometimes the mechanism of injury is known (such as blast exposure or a motor vehicle accident). In these cases, field medics may already have determined the length of LOC, PTA, or other criteria recommended in either the field management guidelines developed by the Brain Trauma Foundation (New York, 
New York) or the Military Acute Concussion Evaluation developed by the DVBIC. These pocket-sized reference cards, in use today, contain a series of questions and symptom checklists that help medics rapidly assess and treat TBI on the battlefield. As time and situations permit, these tests are repeated and neurocognitive testing added (when appropriate) to further define the injury.

In the future, such algorithms and testing tools could be added to handheld devices medics use in the field. Such devices could speed the screening/assessment process and allow forward medical personnel to join TBI symptom data to a patient's medical record. Vital medical history data are already in the electronic dog tags of special operations forces. The Army's Battlefield Medical Information System for Telemedicine is a handheld device that communicates with these dog tags and allows medical personnel to store, retrieve, and transmit data at the point of care [10-11]. As the use of handheld devices becomes more widespread, additional capabilities, such as the TBI guidance and neurocognitive test batteries, could be added to help medical personnel treat wounded soldiers with TBI. The DVBIC is working closely with the Telemedicine and Advanced Technology Research Center (Fort Detrick, Maryland) to integrate such systems into existing devices in theater.

Again, the results of such testing become more relevant when the data can be compared to preinjury performance. Recognizing this fact, the military is moving to collect baseline data on service members prior to deployment. The ability to securely transmit the results of computerized batteries such as the ANAM will be crucial as the Army begins to test individual units prior to deployment and then retest in theater to maintain the health and safety troops on duty.

The VHA is working to determine if similar distancebased systems could be employed to remotely identify cognitive deficits and provide more comprehensive neuropsychological assessment of veteran patients. An ideal system would optimize access without sacrificing reliability, validity, or sensitivity of the measures of important cognitive domains typically impaired following TBI. Unfortunately, no such instruments currently allow delivery via telehealth technology without requiring some sacrifice because of either the assessment environment, patient comfort/familiarity, lack of supervision, or patient motivation and cognitive ability to participate in remote evaluation. Comprehensive neuropsychological assessment requires careful onsite instruction and monitoring.
The VHA recommends further study in this area while it continues to support hybrid approaches of other telehealth forms, such as video consultation and store-andforward technologies. Such techniques may allow distance assessment of patients with TBI while other testing measures are validated that could potentially be used by patients "independently" or with minimal assistance. Promising new approaches are expected to enhance the VA and military response to TBI in the future.

\section{CARE COORDINATION}

Forward medical commands can conduct live and interactive VTC with specialists in the United States. The Army Hospital in Bagram, Afghanistan, for example, uses its VTC system in the operating room for teleneurosurgical mentoring. This system allows general surgeons operating on the patient in theater to consult with specialists at Walter Reed Army Medical Center (WRAMC) (Washington, DC), who are linked into the operation via VTC [3]. These systems are also used widely to enhance care coordination. Interactive VTC connects forward medical units with TBI and polytrauma specialists in the continental United States.

Monthly Department of Defense (DOD)/VA Trauma Continuum of Care VTC meetings provide an opportunity for emergency and surgical staff in theater to communicate with clinical teams worldwide. These meetings focus on critical issues related to polytrauma and the overall evacuation process (Figure). Lead agencies formulate responses to complex medical problems that arise in combat, air transport, and acute care settings. For example, the DVBIC role in this network is to enhance TBI care management for soldiers injured in blasts and motor vehicle accidents and provide recommendations for forward medical units to help identify and treat TBI in the field. Through the use of VTC, clinical and operational concerns can be quickly identified, discussed, and resolved. Equally important, these meetings allow those working directly with wounded soldiers in the field to provide TBI and other trauma specialists with an understanding of the problems related to multiple blast exposure.

The use of VTC continues as individual patients are evacuated to military medical centers such as WRAMC and onward to VA polytrauma centers for subacute care and rehabilitation. Patients treated at WRAMC, for example, are examined by a TBI specialist while recovering 


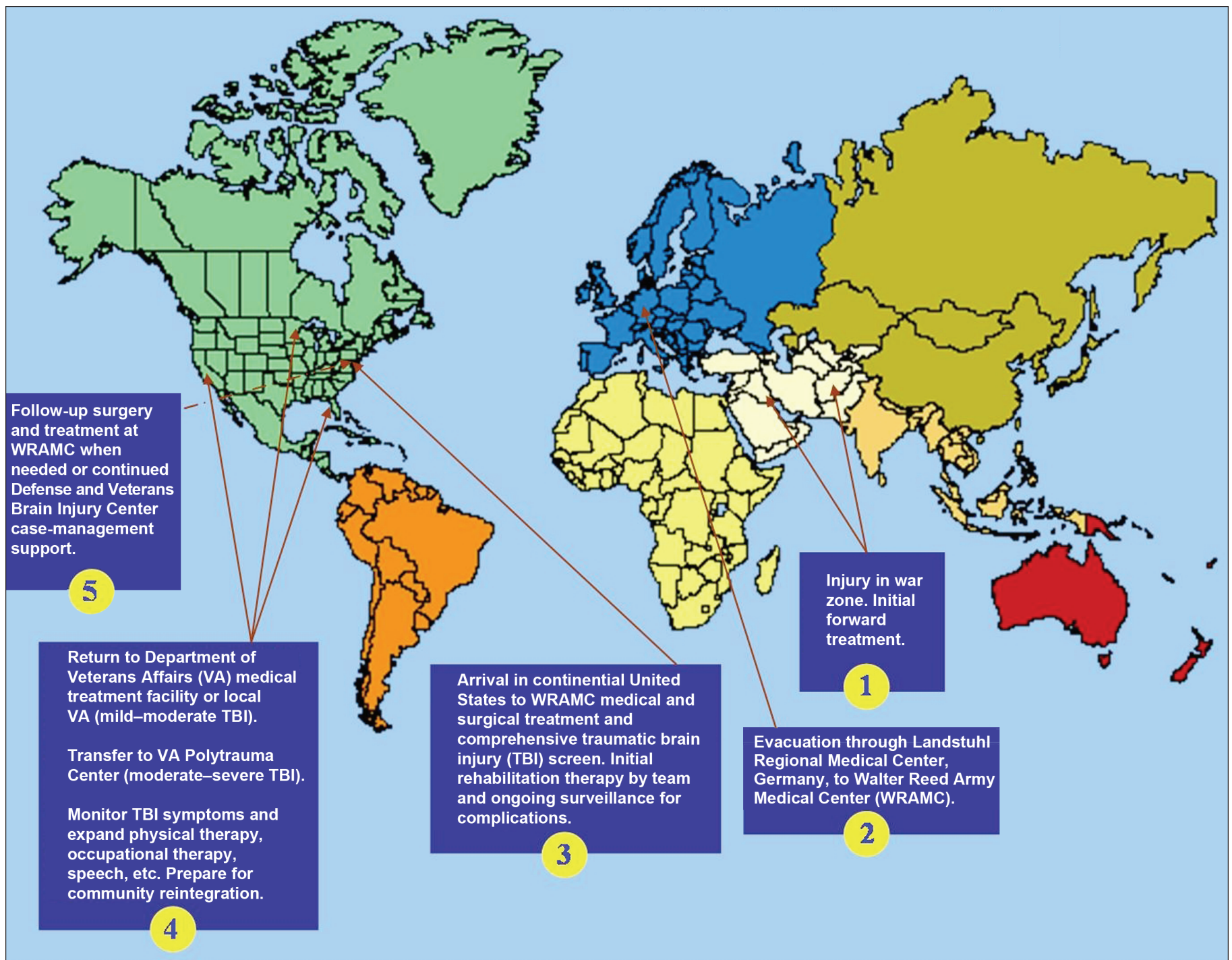

Figure.

Medical evacuation of military patients with traumatic brain injury.

from head and other concomitant injuries. Clinicians at WRAMC meet regularly via VTC with their counterparts at receiving VA TBI polytrauma centers. The goal of these meetings is to ensure continuity of care between sites. Using interactive VTC helps to guide individual patient services and provides a means for patients and family members to prepare for important medical transitions. This added level of coordination is essential for patients with TBI, whose recovery does not always follow a strict linear path. Instead, these patients frequently move back and forth between VA and military medical centers for follow-up surgery, prosthetic adjustments, and other medical treatments as needed.
The VHA Polytrauma Telehealth Network, led by the VHA Office of Care Coordination (OCC) in Washington, DC, takes this concept one step further by establishing interactive connections between clinicians at 4 VA polytrauma centers and 21 Level II VA medical centers (VAMCs) across the country. Transitions to these centers allow service members and veterans to receive necessary care closer to home [12]. The Polytrauma Telehealth Network coordinates national trauma rehabilitation and educational services for patients recovering from traumatic injuries such as TBI, limb amputation, burns, and paralysis. Direct patient evaluation and consultation will also be possible through this expanded VTC network. Together, 
these systems support the entire continuum of care from initial injury to home support, and when possible, return to duty (Figure).

\section{DISTANCE TRAINING FOR PATIENTS AND PROVIDERS}

Practitioners in community settings do not always fully understand the neurobehavioral consequences of brain trauma. Common indicators of mild TBI can easily be mistaken for posttraumatic stress disorder (PTSD), which shares many of the same symptoms as TBI, including fatigue, sleep disturbances, confusion, anxiety, irritability, and decreased concentration. Further, individuals who sustain mild TBI may not present for medical attention. Injured individuals (particularly service members) may prefer to "tough it out" after a concussion, not appreciating that waiting to receive care can increase the likelihood of medical errors and subsequent injury and add psychological distress if they develop PCS without understanding its origin. A challenge in the military, as in civilian life, is ensuring that education is provided to individuals with TBI as well as healthcare providers in community settings. Unfortunately, limited financial resources, time, and distance often prohibit full participation in educational programs.

The healthcare community has long used VTC and Web-based courses for mass dissemination of training and education programs. The TBI community has followed suit, with a host of programs offered through local and national brain injury centers and associations. The National Association of Head Injury Administrators (Bethesda, Maryland) is particularly well known for its ability to direct TBI education to patients and community-based healthcare providers through live and archived Web casts. Events are designed to increase understanding of the various medical, economic, home, and family aspects of living with TBI.

Military and veteran populations face special challenges during TBI treatment and rehabilitation. The complexity of DOD and VA healthcare systems and the cooccurrence of PTSD and other injuries that result from battle can be particularly difficult to manage. Creating a supportive environment of people who can understand these populations as they struggle with conflicting feelings about their combat experience, their military status, and their injuries is critical for their psychosocial healing. With the help of military and VA trauma teams, the DVBIC provides onsite training to help sustain TBI rehabilitation within this unique military context. Basic information in TBI care and recovery can be supplemented by in-depth discussions with experts focusing on difficult issues such as substance abuse, domestic violence, isolation, and depression. Understanding the military culture, health delivery systems, and healing process is a particular asset for TBI specialists, who can convey the practical and psychosocial aspects of brain trauma to providers serving those with combat injuries.

General TBI programs are also available to practitioners who encounter symptoms associated with milder forms of TBI in their clinics. The assistance they receive can help soldiers who may be confused or anxious about their symptoms. Training in TBI identification is particularly meaningful in maintaining unit readiness, because significant numbers of troops return from theater after having been exposed to blasts. To meet the needs of patients and providers who face all levels of TBI severity, the DVBIC combines direct onsite training with interactive VTC and Web-based courses. Clinical workshops on TBI identification and management are offered onsite to medical units throughout the country. Community-based healthcare providers may also earn educational credits by participating in Web-based courses in TBI care management. Interactive VTC allows participants of both "on-site" workshops and Web-based courses to receive follow-up support and guidance as needed.

Planning a broader response to TBI is also possible through technology. A recent multisite event helped to create a complete TBI readiness strategy for communitybased healthcare organizations who serve returning and deploying National Guard troops nationwide. Using VTC, participants at 10 remote military sites interacted with the multidisciplinary team of clinicians during a full-day workshop sponsored by the North Atlantic Medical Command. Follow-up support and direct TBI consultation regarding treatment strategies and operational recommendations are also available through this service.

\section{CLINICAL CONSULTATION VIA VIDEO TELE- CONFERENCING AND WORLD WIDE WEB}

Teleconsultation is among the most frequently used applications of telemedicine today. The American Telemedicine Association (Washington, DC) lists teleconsultation as the principal capability for rendering diagnosis and treatment plans over distance. Teleconsultation can 
involve "provider-to-patient" or "provider-to-provider" interactions that use video, audio, e-mail, or still images with "store-and-forward" technology.

As previously described, the military uses teleconsultation to facilitate higher levels of medical management in the war zone. However, bandwidth shortages in theater can disrupt the high-speed communications necessary for sustaining interactive VTC. So Web-based consultation that uses e-mail and store-and-forward technology is also used. The military's ".consult" program connects clinicians in Iraq and Afghanistan with more than 500 clinical specialists in the United States. Army specialists in dermatology, orthopedics, and internal medicine, for example, can review emergent medical cases and respond to "e-consultations" on a rotating basis [2]. This program supports patients with critical injuries, foreign-born diseases, and complex medical conditions and has allowed hundreds of patients (including Iraqi civilians) to be treated locally. Plans are under way to establish a ".TBI" program that will allow deployed providers to receive immediate expert consultation from brain injury specialists in the United States.

The Army also uses teleconsultation to help manage demand fluctuations at military installations that occur as service members deploy and return in large numbers. The teleneurosurgery program at WRAMC, for example, provides expert neurosurgical consultation to multiple military hospitals in the North Atlantic region. With the assistance of local PAs, patients are encouraged to try conservative treatments for head, neck, and back injuries. These treatments are conducted under the supervision of a distance neurosurgeon who interacts with the patient and local team via VTC. If conservative therapy fails, patients are transferred to WRAMC for neurosurgery and then returned to their duty stations, where continued followup care is provided by their primary care providers onsite and by the distance neurosurgeons via VTC.

A similar teleconsultation program is being formed to address the need for improved TBI identification and management of TBI in service members. This program will augment local resources to meet demand fluctuations for TBI care at troop-intensive sites. A combination of VTC and store-and-forward technology will be used to connect TBI specialists at the Naval Medical Center San Diego (California) with PAs, care managers, and clinicians at military health clinics. A clinical neuropsychologist will be available via VTC to review cognitive tests and consult on neurobehavioral and cognitive issues while a neurologist or other TBI specialist manages the somatic problems of patients with continuing symptoms, also via VTC. A specially trained PA or social worker at each site would work directly with patients to manage symptoms from a total-health perspective. This "virtual TBI clinic" will provide ongoing care for patients with TBI by exploring strategies and appropriate medication management until a positive outcome is achieved.

The changing healthcare needs of veterans have led to similar advances in care coordination for patients with other chronic diseases and conditions, such as diabetes, PTSD, spinal cord injury (SCI), stroke, and multiple sclerosis (MS). Together, the VA's nationwide network of 1,400 facilities and $>250,000$ providers oversees more than 300,000 telemedicine cases annually in 31 clinical specialties [13]. The Care Coordinator General Telehealth Program (Washington, DC) uses VTCs to provide clinical services such as telemental health between hospital- and community-based outpatient clinics. The VA's Care Coordination Store-and-Forward Program likewise makes teleretinal imaging, dermatology, wound care, and pathology services available in rural and remote locations [2]. The VA's regionalized operating structure at the Veterans Integrated Service Network (VISN)-level centralizes these services and provides natural partnerships between tertiary care, rehabilitation, outpatient services, and community support in all parts of the country. Facilities can also connect outside their region for inter-VISN health referrals and specialty consultation.

"The Department of Veterans Affairs is now planning for the large influx of veterans with TBIs from current conflicts who will need continuing care during the coming years" [7]. The ability to provide the "right care at the right time" through teleconsultation is already an important component of the VA's service mix for veterans with TBI. Links between military centers and VA Polytrauma Centers are adding to the VA's ability to provide highly coordinated care for patients with multiple health concerns associated with TBI. These and other telemedicine collaborations in acute settings are leading the way for innovative forms of telemedicine that affect recovery, rehabilitation, and community reentry.

\section{TELEREHABILITATION AND HOME TELEHEALTH FOR PATIENTS WITH TRAUMATIC BRAIN INJURY}

Considerable development has occurred in the areas of telerehabilitation and home telehealth for patients with TBI. Research has shown that a majority of patients with 
TBI, even those recovering from significant brain trauma, can engage with distant providers by using the Internet (with proper assistive technologies) [14]. Additional evidence supports the idea that patients with cognitive impairments can understand the dynamics of interactive VTC and successfully interact with medical professional or loved ones from a distance. Finally, a case study found that teletherapy could be used successfully to improve the functional outcomes, both physical and cognitive, of a patient with severe TBI [15].

Understanding this potential, the VHA OCC recently added brain injury to its clinical areas of focus for telerehabilitation and will be measuring the effects of similar interventions in telerehabilitation programs across the country. Rehabilitation staff at the Minneapolis VAMC (Minnesota), for example, recently supported patients with TBI who were attending college by coordinating the use of personal data assistants (PDAs) that provided necessary memory aids. Other patients were trained to use PDAs to manage their everyday activities, promote independence, and remain in the community. Other areas of focus for the VA telerehabilitation program will include SCI, MS, frail elderly, and such specialty services as speech and audiology.

The growth of home telehealth as an industry has allowed isolated healthcare practitioners in rural communities to receive the advice and consultation of specialists. Home telehealth has also expanded the reach of visiting nurses, who are now able to "check in on" patients from a distance by using the telephone, Internet, and an array of remote monitoring devices that can relay a patient's vital signs and other information to distant healthcare providers in real time.

These systems are increasingly being used to help patients with TBI, who are often isolated by physical disability and social challenges. To prevent veterans from "falling through the cracks," the DVBIC also provides ongoing telephone follow-up of patients with TBI who have returned to their home communities. Regular contact with nurses specializing in TBI can help patients manage lingering physical, emotional, and cognitive symptoms [16-17]. Together with community-based care coordinators, the DVBIC team presents strategies for symptom management, ongoing consultation, counseling, and support to family members. Telephone follow-up can provide consistency during a chronic illness or through the rehabilitation process following other acute events. These interventions help patients develop a sense of mastery over their situation that can be reflected in all aspects of life.

DVBIC researchers are also exploring remote medication management and automated/online response systems to improve the health and safety of patients with TBI, Web-based vocational and educational programs to expand personal opportunities for individuals with TBI, and other forms of home telehealth that have succeeded for patients with TBI and other conditions. The Table depicts some of the emerging telemedicine systems being used to support patients with TBI in military and VA settings [18].

\section{CONCLUSIONS}

The DVBIC treats many of today's combat-wounded soldiers with TBI through clinical programs in military and VAMC nationwide. While the work of these teams is far-reaching, their limited number is insufficient to directly meet the needs of thousands of active-duty military, dependents, and veterans who experience the challenges of TBI in daily life. Therefore, the DVBIC also partners with healthcare providers in local communities, forward medical commands, and other VA and military medical centers to ensure the highest levels of care for patients with TBI. These collaborations can involve extensive training in TBI identification, care management, and coordination, as well as the development of operating systems to support local and regional TBI programs. The DVBIC has developed a multifaceted telemedicine program to help orchestrate these services for service members and veterans with TBI. Because of (1) the growing need for TBI assessment, treatment, and surveillance; (2) the shortage of TBI specialists to meet this need; and (3) the tendency of TBI to place patients at risk for secondary injury, clinical intervention through telemedicine will continue to play an important role in patient care.

\section{ACKNOWLEDGMENTS}

The views expressed in this article are those of the authors and do not necessarily represent the views of the DOD or the VA.

This material is the result of work supported by resources and facilities at the DVBIC, WRAMC, and Manchester VAMC. 
Table.

Telemedicine applications for traumatic brain injury (TBI).

Clinical Domain

TBI Identification

Acute and Subacute Care

Rehabilitation

Home Care

TBI Health Education New or Emerging Paradigm

Healthcare providers use electronic cognitive assessment systems to identify concussion and mild TBI in soldiers after blasts and in athletes after physical contact.

Clinical teams collaborate over distance to coordinate the care of combat-wounded patients with TBI and other critical injuries.

Direct physician consultation, eval- Interactive VTC. uation, and care to patients with TBI and families over distance.

TBI specialists provide distance consultation with forward medics and ER physicians.

Teletherapy for patients with TBI receiving long-term rehabilitation services and nursing care.

Real-time video visits with family members allowing visual communication for patients with severe TBI.

Telephonic and video nursing supervision of patients with TBI in home.

Medication management and automated/online response systems.

Remote cognitive therapy ${ }^{*}$ for patients with TBI

Distance occupation, speech, and physical therapies.

Interactive video programs providing training for medics, physician's assistants, nurses, and other providers in civilian and military settings.

Web courses with certified training Internet. modules. agement devices.

Interactive VTC. information. alert systems.

Internet.

Interactive VTC.

Interactive VTC.
Web-based applications, laptop computers, and handheld data man-

Interactive VTC, multipoint video bridging, and EMR sharing.

EMR systems and e-mail.

Interactive VTC and Web-based management systems.

Telephone, VTC, and medical devices capable of collecting and transmitting vital signs and other

Web-based applications, automated medication dispensers, and

Increased provider knowledge and understanding of TBI symptoms, effects, and relationship to a patient's overall health and independence.

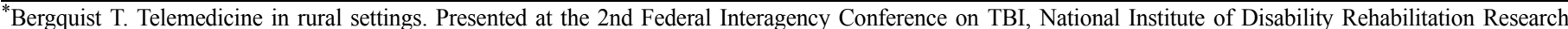
(NIDRR), Mayo Traumatic Brain Injury Model Systems (TBIMS); 2006 Mar 10. Available from: http://www.tbi-interagency.org/conf_presentations.php/ $\mathrm{EMR}=$ electronic medical records, $\mathrm{ER}=$ emergency room, $\mathrm{VTC}=$ video-teleconferencing. 
JRRD, Volume 44, Number 7, 2007

The authors have declared that no competing interests exist.

\section{REFERENCES}

1. Darkins A. Changing the location of care: Management of patients with chronic conditions in Veterans Health Administration using care coordination/home telehealth. J Rehabil Res Dev. 2006;43(4):vii-xii. [PMID: 17123179]

2. Lilie C. Moving images not patients. Signal. 2005 Mar. p. 63.

3. Ackerman RK. Telemedicine reaches far and wide. Signal. 2005 Mar. p. 55-59.

4. The Associated Press. Scientists develop thought-controlled prosthetic limbs: Patients benefit from improved technology. Daily Illini. 2006 Sep 15.

5. White J, Rosenthal M, DeLisa J, editors. Rehabilitation of the patient with brain injury. In: DeLisa JA, editor. Rehabilitation: Principles and practice. 2nd ed. Philadelphia (PA): Lippincott; 1993.

6. Samson K. Increasing Iraq injuries spur demand for rehab services and high-tech research and development. Neurology Today. 2006 Jan 3. p. 21-22.

7. Okie S. Traumatic brain injury in the war zone. N Engl $\mathrm{J}$ Med. 2005;352(20):2043-47. [PMID: 15901856]

8. Collins M. Sports related MTBI: Rates of recovery, risk factors and return to play. Presented at the 2nd Federal Interagency Conference on TBI, University of Pittsburgh Medical Center Sports Concussion Program; 2006 Mar 11; Pittsburgh (PA). Available online from: http://www.tbi-interagency.org/ conf presentations.php/

9. McCrea M, Guskiewicz KM, Marshall SW, Barr W, Randolph C, Cantu RC, Onate JA, Yang J, Kelly JP. Acute effects and recovery time following concussion in collegiate football players: The NCAA Concussion Study. JAMA. 2003;290(19):2556-63. [PMID: 14625332]
10. Kenyon HS. Medics triage from a distance. Signal. 2005 Mar. p. 73-74.

11. Ackerman RK. Dog tags join wireless revolution. Signal. AFCEA. 2005 Mar. p. 58.

12. Cornis-Pop M, Stephens G. VHA polytrauma system of care enhances amputation care for combat injured veterans. Capabilities. 2006;14(3):8-9. Available online from: http:// www.medschool.northwestern.edu/depts/repoc/sectionpublications/capabilities/index.html/

13. VA telemedicine on display. U.S. Medicine. 2002 Jul.

14. Ricker JH, Rosenthal M, Garay E, DeLuca J, Germaine A, Abraham-Fuchs K, Schmidt KU. Telerehabilition needs: A survey of persons with acquired brain injury. J Head Trauma Rehabil. 2002;17(3):242-50. [PMID: 12086577]

15. Forducey PG, Ruwe WD, Dawsom SJ, Scheideman-Miller C, McDonald NB, Hantla MR. Using telerehabilitation to promote TBI recovery and transfer of knowledge. NeuroRehabilitation. 2003;18(2):103-11. [PMID: 12867673]

16. Martin EM, Coyle MK. Nursing protocol for telephonic supervision of clients. Rehabil Nurs. 2006;31(2):54-57. [PMID: 16526522]

17. Bell KR, Temkin NR, Esselman PC, Doctor JN, Bombardier CH, Fraser RT, Hoffman JM, Powell JM, Dikmen S. The effect of a scheduled telephone intervention on outcome after moderate to severe traumatic brain injury: A randomized trial. Arch Phys Med Rehabil. 2005;86(5): 851-56. [PMID: 15895327]

18. Bergquist T. Telemedicine in rural settings. Presented at the 2nd Federal Interagency Conference on TBI, National Institute of Disability Rehabilitation Research (NIDRR), Mayo Traumatic Brain Injury Model Systems (TBIMS); 2006 Mar 10. Available online from: http://www.tbi-interagency.org/conf presentations.php/

Submitted for publication January 10, 2007. Accepted in revised form September 18, 2007. 